PROCEEDINGS OF THE

AMERICAN MATHEMATICAL SOCIETY

Volume 140, Number 9, September 2012, Pages 3041-3047

S 0002-9939(2012)11201-8

Article electronically published on January 30, 2012

\title{
TRANSCENDENTAL VALUES OF CLASS GROUP $L$-FUNCTIONS, II
}

\author{
M. RAM MURTY AND V. KUMAR MURTY \\ (Communicated by Matthew A. Papanikolas)
}

\begin{abstract}
Let $K$ be an imaginary quadratic field and $\mathfrak{f}$ an integral ideal. Denote by $C l(\mathfrak{f})$ the ray class group of $\mathfrak{f}$. For every non-trivial character $\chi$ of $C l(\mathfrak{f})$, we show that $L(1, \chi) / \pi$ is transcendental. If $\mathfrak{f}=\overline{\mathfrak{f}}$, then complex conjugation acts on the character group of $C l(\mathfrak{f})$. Denoting by $\widehat{C l(f)}^{+}$the orbits of the group of characters, we show that the values $L(1, \chi)$ as $\chi$ ranges over elements of $\widehat{C l(\mathfrak{f})}+$ are linearly independent over $\overline{\mathbb{Q}}$. We give applications of this result to the study of transcendental values of Petersson inner products and certain special values of Artin $L$-series attached to dihedral extensions.
\end{abstract}

\section{INTRODUCTION}

Let $K$ be an imaginary quadratic field with discriminant $d_{K}$ and ring of integers $\mathcal{O}_{K}$. Let $\mathfrak{f}$ be an integral ideal of $\mathcal{O}_{K}$ and denote by $C l(\mathfrak{f})$ the ray class group of $\mathcal{O}_{K}$ $(\bmod \mathfrak{f})$, with $K_{\mathfrak{f}}$ the corresponding ray class field over $K$. By class field theory, there is a natural isomorphism

$$
\sigma: C l(\mathfrak{f}) \stackrel{\sim}{\longrightarrow} \operatorname{Gal}\left(K_{\mathfrak{f}} / K\right)
$$

induced by the Artin symbol. For each non-trivial character $\chi$ of $C l(\mathfrak{f})$, the Hecke $L$-series $L(s, \chi)$ extends to an entire function. In this paper, we will investigate the transcendental nature of the numbers $L(1, \chi)$ as $\chi$ ranges over non-trivial characters of $C l(\mathfrak{f})$.

We first consider the case $\mathfrak{f}=\overline{\mathfrak{f}} \neq \mathcal{O}_{K}$. The case when $\mathfrak{f}=\mathcal{O}_{K}$ was dealt with in our earlier paper [3] using different techniques. In this situation also, complex conjugation acts on the ray class group and on the group of ideal class characters. We denote by $C l(\mathfrak{f})^{+}$and $\widehat{C l(\mathfrak{f})}^{+}$, respectively, a set of orbit representatives under this action.

Our main theorem is

Theorem 1. Let $K$ be an imaginary quadratic field and let $\mathfrak{f}$ be an integral ideal of $\mathcal{O}_{K}$ unequal to 1 satisfying $\mathfrak{f}=\overline{\mathfrak{f}}$. As $\chi$ ranges over non-trivial Hecke characters of $\widehat{C l(\mathfrak{f})}^{+}$, the special values $L(1, \chi)$ are linearly independent over $\overline{\mathbb{Q}}$.

Received by the editors July 23, 2010 and, in revised form, March 30, 2011.

2010 Mathematics Subject Classification. Primary 11J81; Secondary 11M32.

Key words and phrases. Class group $L$-functions, transcendental values, Petersson inner products, Artin $L$-series.

The research of both authors was partially supported by NSERC Discovery grants.

(C)2012 American Mathematical Society Reverts to public domain 28 years from publication 
Given a map $f: C l(\mathfrak{f}) \rightarrow \mathbb{C}$, we can define the $L$-series

$$
L(s, f)=\sum_{\mathfrak{a}} \frac{f(\mathfrak{a})}{N(\mathfrak{a})^{s}} .
$$

As in [3], one can show that this extends to an entire function if and only if

$$
\rho_{f}:=\sum_{R \in C l(\mathfrak{f})} f(R)=0 .
$$

In this setting, we can speak of the value $L(1, f)$ and discuss its nature. The consideration of this series can be thought of as the $K$-analogue of the $L$-series introduced by Chowla 2] for the rational number field case (to be discussed in detail below). Since any such function $f$ can be written as a linear combination of characters of $C l(\mathfrak{f})$ and condition (11) implies that only non-trivial characters are involved, it is immediate that we have the following corollary.

Corollary 2. Assume that $\mathfrak{f}=\overline{\mathfrak{f}} \neq \mathcal{O}_{K}$. Let $f: C l(\mathfrak{f}) \rightarrow \overline{\mathbb{Q}}$ be an algebraic valued map not identically zero. Suppose that $\rho_{f}=0$. Then $L(1, f) \neq 0$ unless $f(R)+f\left(R^{-1}\right)=0$ for every ideal class $R \in C l(\mathfrak{f})$.

As remarked above, in an earlier paper [3, we discussed the case $\mathfrak{f}=1$ and proved that the special values $L(1, \chi)$ are linearly independent over $\overline{\mathbb{Q}}$ as $\chi$ ranges over the non-trivial characters of the ideal class group (modulo the action of complex conjugation). The case when $\mathfrak{f} \neq 1$ requires a different treatment than the one used in [3] since the methods there do not directly lead to the results of this paper. The situation when $\mathfrak{f} \neq \overline{\mathfrak{f}}$ is one we relegate to a future paper. However, some of the methods of this paper can be applied to give some information in the general case too. Here are a few results in this direction.

Theorem 3. The special values $L(1, \chi) / \pi$ are transcendental as $\chi$ ranges over nontrivial characters of $\widehat{C l(\mathfrak{f})}^{+}$. Moreover, in the case that $\mathfrak{f}=\overline{\mathfrak{f}}$, there is at most one algebraic number in the list $L(1, \chi), \chi \neq 1, \chi \in \widehat{C l(\mathfrak{f})}^{+}$.

Presumably, $L(1, \chi)$ is always a transcendental number. We are unable to show this. The value can be shown to be equal to $\pi$ times a $\overline{\mathbb{Q}}$-linear form of logarithms of algebraic numbers. Establishing the transcendence of such expressions is, at present, slightly beyond the reach of transcendental number theory.

As will be seen below, the transcendence of $L(1, \chi)$ is a consequence of Schanuel's conjecture. This conjecture states that if $x_{1}, \ldots, x_{n}$ are linearly independent numbers over $\mathbb{Q}$, then the transcendence degree of the field

$$
\mathbb{Q}\left(x_{1}, \ldots, x_{n}, e^{x_{1}}, \ldots, e^{x_{n}}\right)
$$

is at least $n$. Although some progress has been made on some special cases of this conjecture, it is far from being proved.

However, what we need is a "weaker" version of this conjecture. Baker's theorem asserts that if $\alpha_{1}, \ldots, \alpha_{n}$ are algebraic numbers so that

$$
\log \alpha_{1}, \ldots, \log \alpha_{n}
$$

are linearly independent over $\mathbb{Q}$, then they are linearly independent over $\overline{\mathbb{Q}}$. Schanuel's conjecture implies that

$$
\log \alpha_{1}, \ldots, \log \alpha_{n}
$$


are algebraically independent if they are linearly independent over $\mathbb{Q}$. It is this assertion (or more precisely, a weaker version of it) that is needed to deduce that $L(1, \chi)$ is itself transcendental whenever $\chi$ is a character of the ray class group of $K$.

These results have several applications. The first is to establish transcendence of certain Petersson inner products. The second is to special values of Artin $L$-series attached to $S_{3}$ extensions of $\mathbb{Q}$. We state these results below.

Theorem 4. Let $K$ be an imaginary quadratic field and $\mathfrak{f}$ an ideal of the ring of integers of $K$. For each non-trivial character $\chi$ of $C l(\mathfrak{f})$, let $f_{\chi}$ be the classical normalized Hecke eigenform such that $L(s, \chi)=L\left(s, f_{\chi}\right)$. Suppose that $\chi^{2} \neq 1$. The Petersson inner products $\left(f_{\chi}, f_{\chi}\right) / \pi$ are transcendental numbers. Moreover, there is at most one algebraic number in the list $\left(f_{\chi}, f_{\chi}\right)$ as $\chi$ ranges over non-trivial characters in $\widehat{C l(\mathfrak{f})}^{+}$with $\chi^{2} \neq 1$.

We record the following application to special values of certain non-abelian $L$ series.

Theorem 5. Let $M / \mathbb{Q}$ be a Galois extension with Galois group $D_{2 n}$, the dihedral group of order $2 n$. Let $\psi$ be an irreducible character of $D_{2 n}$ of degree $\neq 1$ and $L(s, \psi)$ the Artin L-series attached to $\psi$. Then $L(1, \psi) / \pi$ is transcendental.

\section{Group-theoretic Preliminaries}

We begin with a straightforward result from group theory which is an interesting variant of Artin's theorem on the linear independence of the irreducible characters of a finite group.

Lemma 6. Let $G$ be a finite group. Suppose that

$$
\sum_{\chi \neq 1} \chi(R) u_{\chi}=0
$$

for all $R \neq 1$ and all irreducible characters $\chi \neq 1$ of $G$. Then $u_{\chi}=0$ for all $\chi$.

Proof. This is Lemma 3 of [4] and is easily deduced using the orthogonality relations.

\section{A REVIEW OF RAMACHANDRA INVARIANTS AND BAKER'S THEORY}

In this section, we recall and record several results due to Ramachandra [7] that will be needed in the proof. Since $\mathfrak{f}=\overline{\mathfrak{f}}$, complex conjugation acts on $C l(\mathfrak{f})$ and we denote by $C l(\mathfrak{f})^{+}$the equivalence classes of ideal classes under this action. We also write $\Re(z)$ for the real part of a complex number $z$. Since $C l(\mathfrak{f})$ is a finite group, the character values are roots of unity.

Lemma 7. Let $\mathfrak{f} \neq 1$ and suppose that $\mathfrak{f}=\overline{\mathfrak{f}}$. There exist non-zero algebraic numbers $A_{\chi}$ and multiplicatively independent units $\epsilon(R)\left(R \in C l(\mathfrak{f})^{+}, R \neq 1\right)$ such that

$$
L(1, \chi)=\pi A_{\chi} \sum_{R \neq 1, R \in C l(\mathfrak{f})^{+}} \delta_{R} \Re(\chi(R)) \log |\epsilon(R)|
$$

(with $\delta_{R}=1$ if $R^{2}=1$ and 2 otherwise) for every non-trivial character $\chi$ of $C l(\mathfrak{f})$. Moreover, the formula implies $\overline{A_{\chi}}=A_{\bar{\chi}}$. If $\mathfrak{f} \neq 1$ and $\mathfrak{f} \neq \overline{\mathfrak{f}}$, then $L(1, \chi) / \pi$ is a $\overline{\mathbb{Q}}$-linear form in logarithms of algebraic numbers. 
Proof. This is a direct consequence of Theorems 8, 9 and 12 of [7]. In particular, the corollary on page 134 of [7] gives the multiplicative independence of the units.

In the discussion below, a pivotal role is played by the fundamental theorem of Baker concerning linear forms in logarithms. We record this as

Lemma 8. If $\alpha_{1}, \ldots, \alpha_{n} \in \overline{\mathbb{Q}} \backslash\{0\}$ and $\beta_{1}, \ldots, \beta_{n} \in \overline{\mathbb{Q}}$, then

$$
\beta_{1} \log \alpha_{1}+\cdots+\beta_{n} \log \alpha_{n}
$$

is either zero or transcendental. The latter case arises if $\log \alpha_{1}, \ldots, \log \alpha_{n}$ are linearly independent over $\mathbb{Q}$ and $\beta_{1}, \ldots, \beta_{n}$ are not all zero.

Proof. This is the content of Theorems 2.1 and 2.2 of [1]. Let us note that here and later we interpret log as the principal value of the logarithm with the argument lying in the interval $(-\pi, \pi]$.

In particular, if $\log \alpha_{1}, \ldots, \log \alpha_{n}$ are linearly independent over $\mathbb{Q}$, then they are linearly independent over $\overline{\mathbb{Q}}$.

Thus, an immediate application of Baker's theory of linear forms in logarithms leads to two corollaries in our context. The first is that the numbers $\log |\epsilon(R)|$ as $R$ ranges over non-trivial elements of $C l(\mathfrak{f})^{+}$are linearly independent over $\overline{\mathbb{Q}}$ since they are linearly independent over $\mathbb{Q}$. The second is that $L(1, \chi) / \pi$ is transcendental for every non-trivial character $\chi$ of $C l(\mathfrak{f})$.

As a further application of Lemma 8 we prove the following variant of a result from [5].

Lemma 9. Let $\alpha_{1}, \alpha_{2}, \ldots, \alpha_{n}$ be positive algebraic numbers. If $c_{0}, c_{1}, \ldots, c_{n}$ are algebraic numbers with $c_{0} \neq 0$, then

$$
c_{0} \pi+\sum_{j=1}^{n} c_{j} \log \alpha_{j}
$$

is a transcendental number and hence non-zero.

Proof. This is Lemma 9 of [4] and is easily deduced from Baker's theorem.

\section{Proof of Theorem 1}

Suppose that

$$
\sum_{\chi \neq 1, \chi \in \widehat{C l(\mathfrak{f})}} c_{\chi} L(1, \chi)=0
$$

By Lemma 7, we have

$$
L(1, \chi)=\pi A_{\chi} \sum_{R \in C l(\mathfrak{f})^{+}, R \neq 1} \delta_{R} \Re(\chi(R)) \log |\epsilon(R)|,
$$

where $A_{\chi}$ is a non-zero algebraic number. Hence, inserting this into our previous formula,

$$
\sum_{R \in C l(\mathfrak{f})^{+}, R \neq 1} \delta_{R}(\log |\epsilon(R)|)\left(\sum_{\chi \neq 1} \Re(\chi(R)) c_{\chi} A_{\chi}\right)=0 .
$$


Since the $\log |\epsilon(R)|\left(R \in C l(\mathfrak{f})^{+}, R \neq 1\right)$ are linearly independent over $\mathbb{Q}$, by Baker's theorem, they are linearly independent over $\overline{\mathbb{Q}}$, and we deduce that

$$
\sum_{\chi \neq 1} \Re(\chi(R)) c_{\chi} A_{\chi}=0
$$

for every $R \neq 1, R \in C l(\mathfrak{f})^{+}$. Thus, for every $R \neq 1$,

$$
\sum_{\chi \neq 1} \chi(R)\left(c_{\chi} A_{\chi}+c_{\bar{\chi}} A_{\bar{\chi}}\right)=0 .
$$

By Lemma 6, we have $c_{\chi} A_{\chi}+c_{\bar{\chi}} A_{\bar{\chi}}=0$. Since $A_{\chi} \neq 0$, and $\overline{A_{\chi}}=A_{\bar{\chi}}$ and $c_{\chi}=c_{\bar{\chi}}$, we conclude that $c_{\chi}=0$ for every $\chi \neq 1$. This concludes the proof.

\section{Proof of Theorem 3}

As already noted in Lemma $7, L(1, \chi) / \pi$ is a non-vanishing $\overline{\mathbb{Q}}$-linear form in logarithms of algebraic numbers. By Baker's theorem, it is transcendental. Suppose now that $\mathfrak{f}=\overline{\mathfrak{f}}$ and $L\left(1, \chi_{1}\right)=\alpha, L\left(1, \chi_{2}\right)=\beta$ are both algebraic for two distinct Hecke characters $\chi_{1}, \chi_{2}$ of $\widehat{C l(\mathfrak{f})}^{+}$. Let $f=\beta \chi_{1}-\alpha \chi_{2}$. Then $L(1, f)=0$. But by Corollary 2, this implies $f$ is identically zero. By the linear independence of characters, we get $\chi_{1}=\chi_{2}$, a contradiction. Hence, there is at most one character $\chi$ of $\widehat{C l(\mathfrak{f})}^{+}$such that $L(1, \chi)$ is algebraic.

\section{Proof of Theorem 4}

Before we embark on the proof of Theorem 4 , we discuss briefly the relevant portion of Rankin-Selberg theory. If $f$ is a Hecke eigenform of weight $k$ and level $N$, there is an automorphic representation $\pi_{f}$ of $G L_{2}\left(A_{\mathbb{Q}}\right)$ corresponding to $f$ such that

$$
L\left(s, \pi_{f}\right)=L(s+k-1, f) .
$$

If $k=1$ (as it will be in the case of interest to us), the factor corresponding to the prime $p$ (with $p$ coprime to $N$ ) in the Euler product of $L(s, f)$ is of the form

$$
\left(1-\frac{\alpha_{p}}{p^{s}}\right)^{-1}\left(1-\frac{\beta_{p}}{p^{s}}\right)^{-1}
$$

with $\left|\alpha_{p}\right|=\left|\beta_{p}\right|=1$. For primes $p \mid N$, the Euler factor is of the form

$$
\left(1-\frac{\alpha_{p}}{p^{s}}\right)^{-1}
$$

with $\alpha_{p}$ algebraic. The factor corresponding to the prime $p$ (with $(p, N)=1$ ) in the Euler product of the symmetric square $L$-function of $f L\left(s, \operatorname{Sym}^{2}\left(\pi_{f}\right)\right)$ is

$$
\left(1-\frac{\alpha_{p}^{2}}{p^{s}}\right)^{-1}\left(1-\frac{\alpha_{p} \beta_{p}}{p^{s}}\right)^{-1}\left(1-\frac{\beta_{p}^{2}}{p^{s}}\right)^{-1} .
$$

The Euler factor for $p \mid N$ is of the form $F\left(p^{-s}\right)^{-1}$ with $F$ a polynomial of degree at most 3 and with algebraic coefficients, satisfying $F(0)=1$.

If $\chi$ is a non-trivial character of $C l(\mathfrak{f})$, then there is a normalized Hecke eigenform $f_{\chi}$ of level $N_{\chi}$ and Nebentypus $\epsilon$ such that $L\left(s, f_{\chi}\right)=L(s, \chi)$. For primes $p$ coprime 
to $N_{\chi}$, the $\alpha_{p}, \beta_{p}$ can be described explicitly in terms of $\chi$ as follows. If $p$ is inert in $K$, then

$$
\left(1-\alpha_{p} T\right)\left(1-\beta_{p} T\right)=1-\chi((p)) T^{2} .
$$

If $p$ splits as $(p)=\mathfrak{p p}^{\prime}$, then

$$
\left(1-\alpha_{p} T\right)\left(1-\beta_{p} T\right)=(1-\chi(\mathfrak{p}) T)\left(1-\chi\left(\mathfrak{p}^{\prime}\right) T\right) .
$$

Using the fact that $\alpha_{p} \beta_{p}=\epsilon(p)$ and writing $u \sim v$ to mean that $u / v$ is algebraic, it is not difficult to see that

$$
L\left(2, \epsilon^{2}\right) L\left(1, \chi^{2}\right) \sim L\left(1, \operatorname{Sym}^{2}\left(\pi_{f}\right)\right) .
$$

Since $\epsilon^{2}$ is even, $L\left(2, \epsilon^{2}\right)$ is an algebraic multiple of $\pi^{2}$ (see, for example, 6]). Writing $a \sim b$ if $a / b$ is an algebraic number, we have on the other hand, $L\left(1, \operatorname{Sym}^{2}\left(\pi_{f}\right)\right) \sim$ $(f, f) \pi^{2}$ from well-known formulas (see, for example, 9]). It follows that $(f, f) \sim$ $L\left(1, \chi^{2}\right)$.

Although we verified this for $p$ coprime to $N_{\chi}$, the result is valid for all $p$, though this is not essential for the proof of Theorem 4 since the finite number of Euler factors introduce only a non-zero algebraic factor in the final evaluation of $L\left(1, \chi^{2}\right)$.

Thus,

$$
(f, f) \sim L\left(1, \chi^{2}\right)
$$

Our theorem implies that $(f, f) / \pi$ is transcendental, being a non-vanishing $\overline{\mathbb{Q}}$-linear form in logarithms of algebraic numbers. Again, if we list these modular forms $f_{i}$ arising in this way from Hecke characters $\widehat{C l(\mathfrak{f})}^{+}$, then there are at most two algebraic values of the inner products in this list. The proof is analogous to the one given above for Theorem 3 . This completes the proof of Theorem 4 .

\section{Proof of Theorem 5}

By familiar group theory, $\psi$ is induced from a character $\tau$ of the cyclic subgroup $C_{n}$ of order $n$ (see, for example, pp. 37-38 of [8]). If we let $K$ be the subfield fixed by $C_{n}$, then $K$ is a quadratic extension of $\mathbb{Q}$. By properties of Artin $L$-series, $L(s, \psi)=L(s, \tau)$ where the latter is the Artin $L$-series attached to $\tau$ corresponding to the abelian extension $M / K$. By Artin's reciprocity law, there is a Hecke character $\chi$ so that $L(s, \tau)=L(s, \chi)$. If $K$ is an imaginary quadratic field, then by Theorem 3 , we have that $L(1, \chi) / \pi$ is transcendental. If $K$ is a real quadratic field, then $L(1, \chi)$ is a non-zero algebraic multiple of $\pi^{2}$ (see p. 133 of [10]). In both cases, the assertion of the theorem is established.

\section{Concluding Remarks}

One can ask if similar results can be obtained for other algebraic number fields. If $K$ is a real quadratic field, then the result does not hold since, in this case, one can show that $L(1, \chi)$ is an algebraic multiple of $\pi^{2}$ (see, for example, [10]). Thus, some caution is needed in formulating a general conjecture, and we relegate this to future work.

\section{ACKNowledgement}

We thank the referee for helpful remarks on an earlier version of this paper. 


\section{REFERENCES}

[1] A. Baker, Transcendental Number Theory, Cambridge University Press, 1975. MR 0422171 (54:10163)

[2] S. Chowla, The nonexistence of nontrivial linear relations between roots of a certain irreducible equation, Journal of Number Theory, 2 (1970), 120-123. MR0249393 (40:2638)

[3] M. Ram Murty and V. Kumar Murty, Transcendental values of class group $L$-functions, to appear in Math. Annalen. (Published online 12 December 2010.) DOI:10.1007/S00208-0100619-y

[4] M. Ram Murty and V. Kumar Murty, A problem of Chowla revisited, Journal of Number Theory, 131 (2011), no. 9, 1723-1733. MR2802143

[5] M. Ram Murty and N. Saradha, Euler-Lehmer constants and a conjecture of Erdös, Journal of Number Theory, 130 (2010), no. 12, 2671-2682. MR2684489

[6] M. Ram Murty and N. Saradha, Special values of the polygamma functions, International Journal of Number Theory, 5, No. 2 (2009), 257-270. MR2502808 (2010a:11143)

[7] K. Ramachandra, Some applications of Kronecker's limit formulas, Annals of Mathematics, 80 (2) (1964), 104-148. MR0164950 (29:2241)

[8] J.-P. Serre, Linear representations of finite groups, Springer-Verlag, New York, 1977. MR0450380 (56:8675)

[9] G. Shimura, The special values of the zeta functions associated with cusp forms, Comm. Pure Appl. Math., 29 (1976), no. 6, 783-804. MR.0434962 (55:7925)

[10] C. L. Siegel, Advanced Analytic Number Theory, TIFR, Bombay, 1980. MR659851 (83m:10001)

Department of Mathematics, Queen's University, Kingston, Ontario, K7L 3N6, CANADA

E-mail address: murty@mast.queensu.ca

Department of Mathematics, University of Toronto, Toronto, Ontario, M5S 2E4, CANADA

E-mail address: murty@math.toronto.edu 